\title{
Using Image Processing for Flame Diagnostics
}

\author{
Joachim Lundberg $^{1} \quad$ Mathias Henriksen $^{1} \quad$ André Gaathaug $^{1}$ \\ ${ }^{1}$ Department of process, energy and environmental technology, University College of Southeast Norway, Norway, \\ Joachim. lundberg@usn. no
}

\begin{abstract}
An image processing routine was developed to determine the flame height of a hydrogen jet flame. Two different techniques was tested, where one where based on Wiens distribution of a black body and one based on intensity of the flame. The method based on intensity gave the best results.
\end{abstract}

Keywords: Image Processing, Hydrogen jet, Wiens distribution, Experimental, high-speed imaging.

\section{Introduction}

Due to both practical and economic reasons, image processing can be a valuable technique for determine flame diagnostics. This study uses image processing to determine the height of a hydrogen jet flame.

A hydrogen jet flame is a diffusion flame as shown in Figure 1. The fuel, and in this case hydrogen, flows from a nozzle or an orifice. On the surface of the jet, the fuel will meet the oxidizer (air/oxygen) and make up the reaction zone or flame. This thin layer will position itself where the mixture of fuel and oxidizer is stoichiometric and the temperature will be about $2000^{\circ} \mathrm{C}$. Since the jet flame is a spatial phenomenon, the flame will be visible all around the jet flame.

Direct measurements of physical properties of fires can be demanding to conduct. Often the high temperature of the fire can harm the measurement equipment or the measurement equipment itself can affect the measurements to give faulty results.

Image based techniques have an advantage in nonintrusiveness and take the measurement equipment away from the fire. This assures the results to be representative for the fire.

In the literature, several flame/fire diagnostic methods is provided using laser based techniques such as PLIF (planar laser induced fluoresce). This technique can provide information of the shape/thickness/position of the flame and reaction zone. The disadvantage with this technique is however the price of the equipment and the robustness of the measurements. Often, soot and particles of a fire can interfere with the measurement equipment to make the usage of PLIF demanding.

In a study by (Trindade et al, 2014) a normal color CCD camera was used to find the air/fuel ratio of a methane and propane flame. The color RGB images was experimentally observed to provide information of the flame.

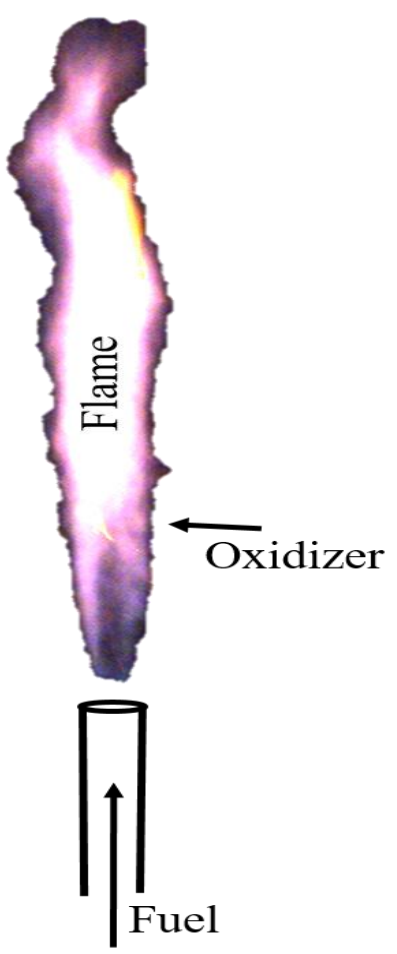

Figure 1. Example of a jet flame

In studies by (Li et al, 2011 and Yan et al., 2017) an image processing routine was developed to estimate temperatures in coal-fired boilers.

These studies base their image processing codes on Wiens distribution law. It assumes black body radiation from the coal or soot particles to be dominating the radiation in the flame.

Alternatively, a model based on intensity and visual considerations of the flame could be used. The model relates the intensity of the flame to the position of the flame.

In a study by Mogi et al. (2005) the flame length and diameter of a hydrogen jet was measured. To visualize the flame, a salt-solution was added to the flame as atomized droplets.

In this work, a hydrogen flame was investigated. It radiated much less than a comparable hydrocarbon or coal flame, but by visual considerations the flame radiated yellow/orange. This might be due to impurities in the hydrogen, but the radiation of the flame was enhanced. The aim of this study was to compare the two methods and find a method to determine the flame height of a hydrogen flame using image processing. 


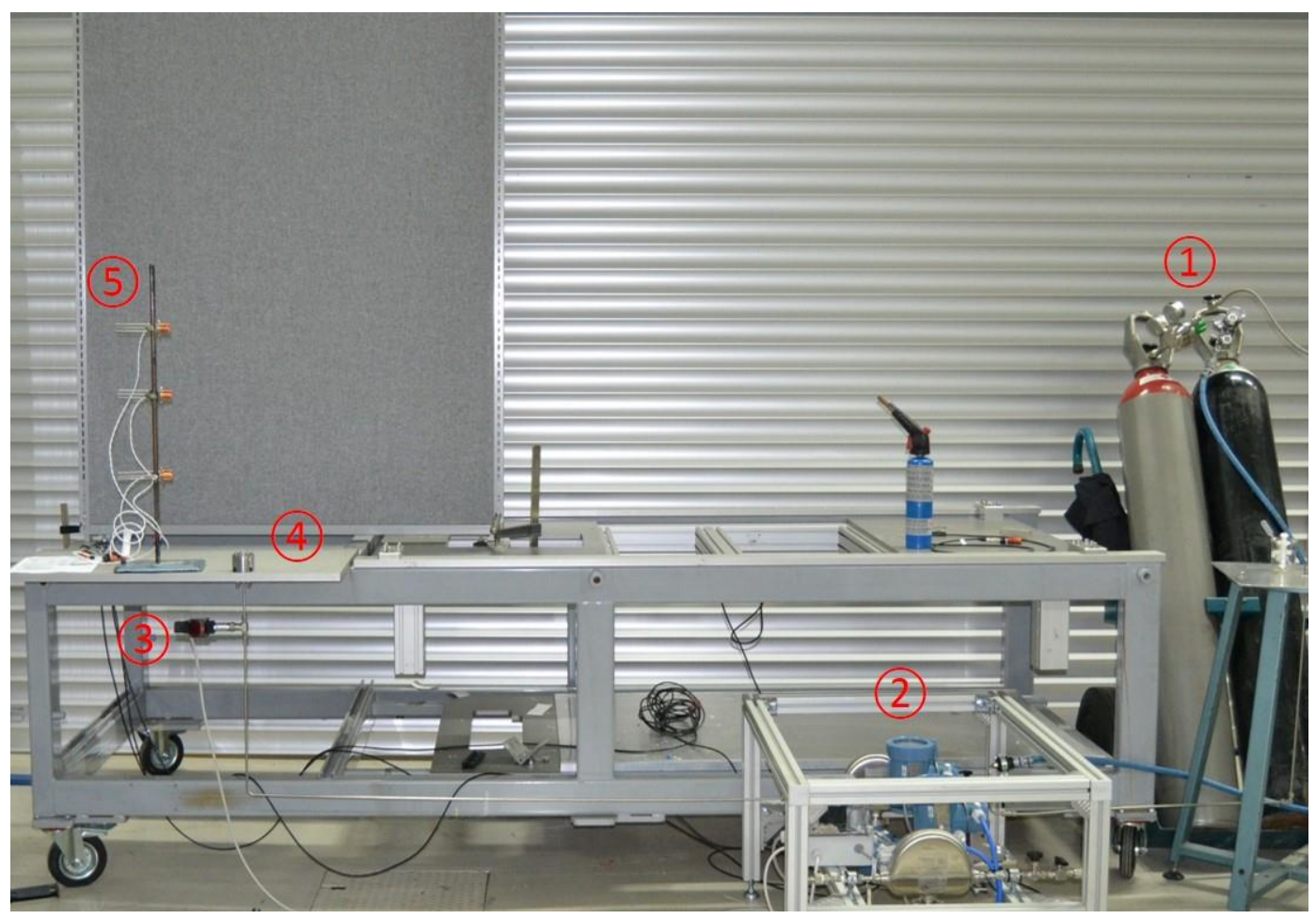

Figure 2. The experimental setup: 1) Hydrogen gas cylinder, 2) mass flow meter, 3) pressure sensor, 4) nozzle unit with changeable nozzle configurations, 5) radiation sensors (currently not in use).

An experimental setup was used with flow and pressure sensors. The images of the hydrogen flame was captured with a color CMOS high-speed camera. The property extracted from the images by an image processing techniques was flame height. The flames investigated, was a hydrogen flame in the range 24 to $1027 \mathrm{~kW}$ (based on lower heating value).

MATLAB was used as a platform for image processing. The purpose of the code was to find the flame height and diameter automatically from the images. The code had the possibility to find the frame height and diameter in every image of the movie, not just the average height.

\section{Experimental Setup}

The experimental setup is shown in Figure 2. The experimental setup was supplied with compressed hydrogen at $20 \mathrm{MPa}$ from a cylinder and pressure controlled by a pressure regulator with a range from 1.0 to $12 \mathrm{MPa}$. A Coriolis mass flow meter from MicroMotion was used to accurately measure the mass flow of hydrogen. A pressure sensor close to the nozzle was mounted to get the pressure before the nozzle. The nozzle unit with interchangeable nozzles was mounted on an aluminum table. A row of radiation sensors was mounted for future use.

The flame was captured using a color high-speed camera with frame rate of 250 frames per second (fps). The data from the logging instruments, such as mass flow meter, pressure sensors etc, was synchronized with the camera and recorded at $2000 \mathrm{~Hz}$. Each experiment had a duration of 5 seconds after the system had stabilized, which took approximately 2-4 seconds after ignition. For each experiment, 1250 images and 9999 data points for each of the other sensors was stored. Afterwards the image was analyzed with an in-house developed image-processing tool to determine the flame height. The flame height was determined to be from the nozzle exit (top of the nozzle) to the tip of the flame.

\section{Choice of Method}

The key feature of the image processing code was to determine the flame in high speed images to find the flame height of a hydrogen flame. Two methods was considered for this task: One based on Wiens distribution and one on image intensity.

The method that used Wiens distribution by (Huang et al., 2000) was an image based method to monitor the temperature of a 500-kW model furnace with both coal and gas as a fuel. The temperature of the flame was found by relating the intensity of two separate wavelengths from the flame using the expression:

$$
T=\frac{C_{2}\left(\frac{1}{\lambda_{2}}-\frac{1}{\lambda_{1}}\right)}{\left[\ln \frac{G\left(\lambda_{1}, T\right)}{G\left(\lambda_{2}, t\right)}+\ln \frac{S_{\lambda 2}}{S_{\lambda 1}}+\ln \left(\frac{\lambda_{1}}{\lambda_{2}}\right)^{6}\right]}
$$

where $T$ is the temperature, $C_{2}$ is a constant, $\lambda_{1}$ and $\lambda_{2}$ is the first and second wavelength, $G\left(\lambda_{i}, T\right)$ is the measured grayscale value of each wavelength and $S\left(\lambda_{i}\right)$ is the spectral sensitivity of the sensor for the respectively wavelength. The measured grayscale 
values was found by using two spectral filters. One monochrome camera was used to capture the two wavelengths using quick temporal shifting of the filters. In a hydrogen diffusion flame the temperature can reach more than $2000^{\circ} \mathrm{C}$. This made the gasses radiate. If the flame was a sooting flame, most of the soot will radiate as a solid, and be combusted in the flame. When observing a diffusion flame with a digital camera the radiation can be observed as an intensity. The digital image will have an elevated intensity where the flame occurs.

(Zhang \& Hui, 2011) has performed a study on 200MW boiler. They capture digital images of a flame and remove the background of the image, then threshold the image by intensity to find the outline of the flame.

In this study, a hydrogen diffusion jet flame was investigated. A hydrogen jet is radiating considerably less than a coal or hydrocarbon flame. Figure 3a shows the intensity scaled image of the hydrogen jet. The actual image of the flame is in Figure $3 \mathrm{~b}$. The radiation from the flame in very low, which was expected from a hydrogen flame.

To identify the flame in the image of the hydrogen flame, the temperature could be used; The temperature in the diffusion flame will be at the highest where the stoichiometry equals unity. In an image, the flame will be a "shell" on the hydrogen jet hence a constant high temperature from one side of the flame to the other. This is demonstrated in Figure 3a.

Equation 1 can be used to find the temperature in a flame based on images. In this study it was not important to determine the temperature, just the position of the flame. Since the value of the temperature was irrelevant, the equation can be simplified. The wavelengths $\lambda_{1}$ and $\lambda_{2}$ is the green and red part of the original RGB image. Both of these parts will capture a range of wavelengths, but was assumed to have just one. The spectral sensitivity of the sensor was assumed to be equal for the two wavelengths. By inserting this into the Equation 1, the correlation in Equation 2 is found.

$$
T \propto C_{1} /\left[\ln \frac{G\left(\lambda_{1}, t\right)}{G\left(\lambda_{2}, t\right)}+C_{2}\right]
$$

The constants $\mathrm{C} 1$ and $\mathrm{C} 2$ was arbitrarily.

In Figure 4 the values of the different RGB colors from a line through the flame was plotted without amplification. It shows the different channels to be fairly equally shaped. This made the assumption of a Wiens distribution false.

The light sensitivity of the high speed camera can be fairly high, but due to the high repetition rate the light level of each image will be low. In the experiments on the hydrogen jet the maximum light intensity was about $3 \%$ of the maximum obtainable on the sensor. This will make the images highly sensitive to noise.

Figure 5 shows a histogram of all the values of the pixels in the image.
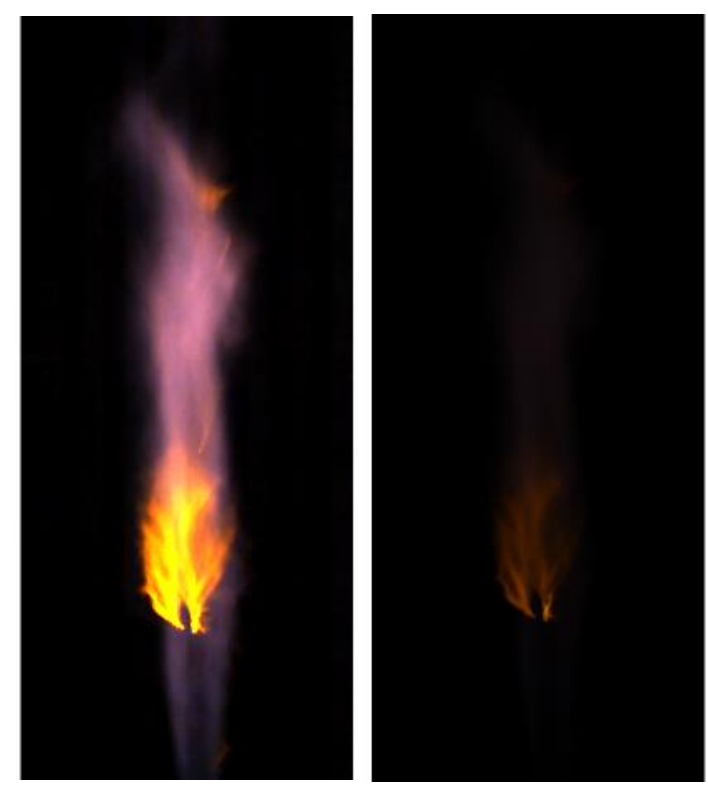

Figure 3. a)Scaled image of hydrogen jet. b) Original image of hydrogen jet

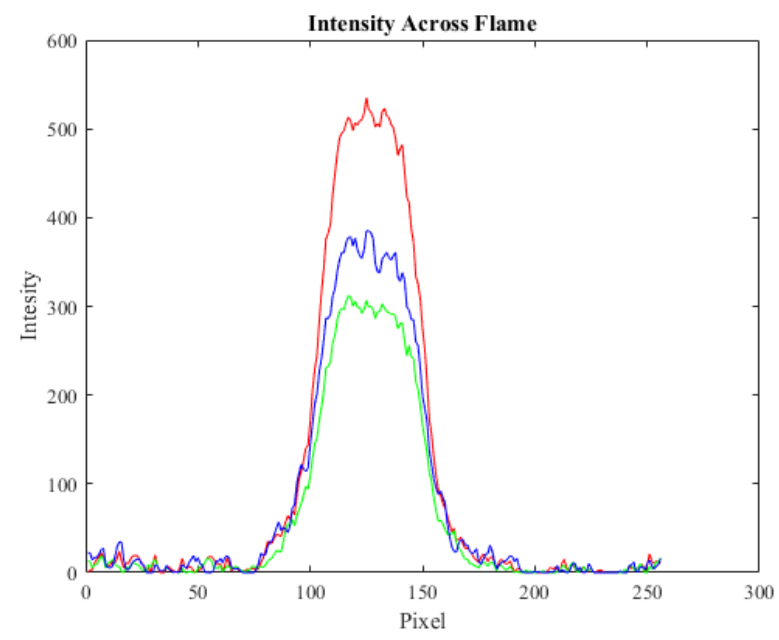

Figure 4. Intensity of a line profile of a horizontal line through the flame.

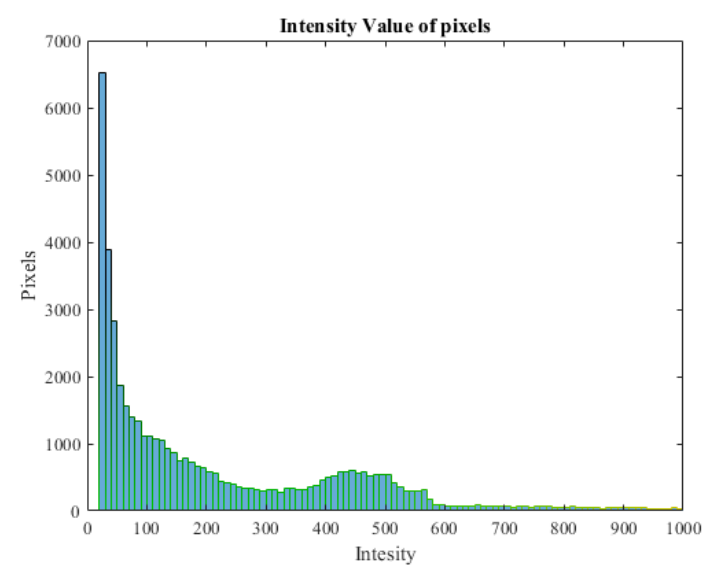

Figure 5. Histogram of pixel values in an image of a hydrogen jet.

As a comparison, the same technique was used on an image of a hydrocarbon fire shown in Figure 6. The 
same image was processed with an expression like Equation 2 shown in Figure 7. Mogi et al. (2005) calculated the emissivity coefficient of a hydrogen flame to be $\epsilon=0.03$ which is fairly low.

During the experiments on the hydrogen jet, an infrared camera was tested to see the temperature distribution in the flame. Figure 8 shows the infrared image from one of the experiments with the hydrogen jet flame. It shows a clear temperature distribution in the flame. Unfortunately, the infrared imaging has limited temporal resolution that will smoothen the shape of the flame.

Instead of using some expression based on Wiens distribution, flame intensity in the image was used. Where the image intensity was high, the flame was present.

Since the image was a color image, the image was separated in three monochrome images, one for each color channel (red, green, blue). This is shown in Figure 9. The three color channels to shows be almost identical in shape and value. Due to this, the red channel was chosen to represent the intensity of the image. The framework to isolate the flame and measure the flame height was developed in a MATLAB script.

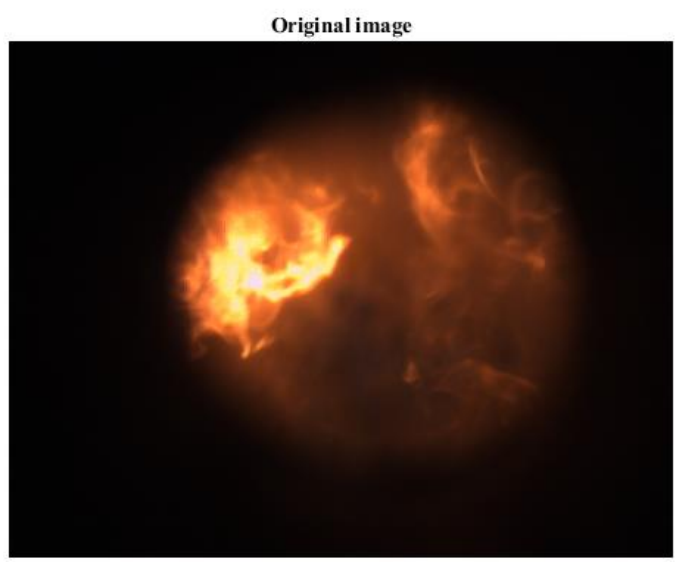

Figure 6. Original image of a hydrocarbon flame.

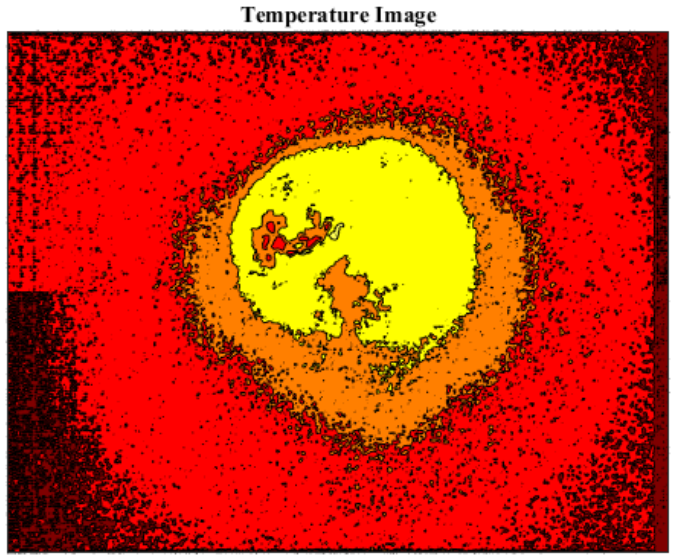

Figure 7. Calculate image of a hydrocarbon flame based on Wiens distribution.

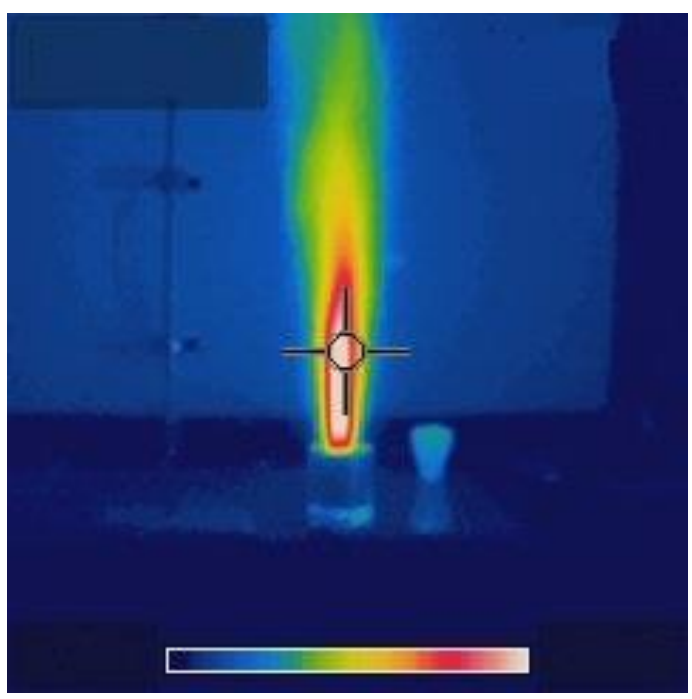

Figure 8. Infra red image of hydrogen jet flame
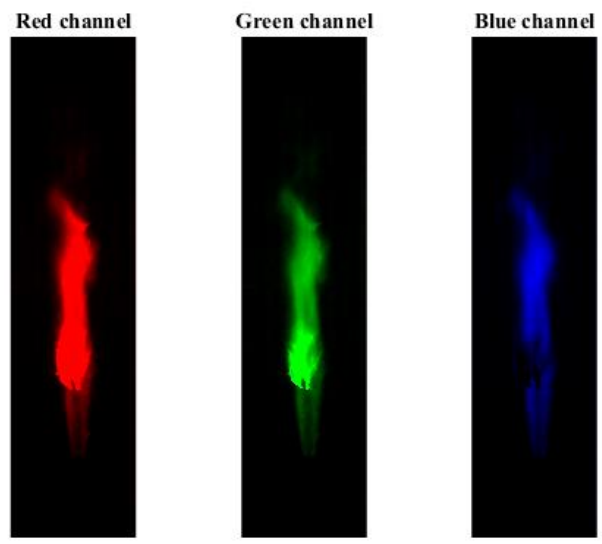

Figure 9. RGB-Color channels of an image of a hydrogen jet flame

\section{Image Processing}

To transfer each image from the movie of the hydrogen flame to a numerical value, an image-processing tool was developed in MATLAB.

Thresholding the image was the most crucial part of the image processing. The threshold value was the set point which distinguished background noise from signal. Background noise in the image could be mistaken for a flame if the procedure for thresholding was to crude. Thru several tests with manual/visual verification, the procedure for thresholding showed good correlation between manually finding the flame height in the images.

To reduce the computation time only the red image was analyzed for the whole data set, but it was thoroughly investigated that each color gave close to identical flame heights. The red color was chosen above the other colors since it had a higher intensity.

The noise in the background of the images was assumed to be normal distributed. 

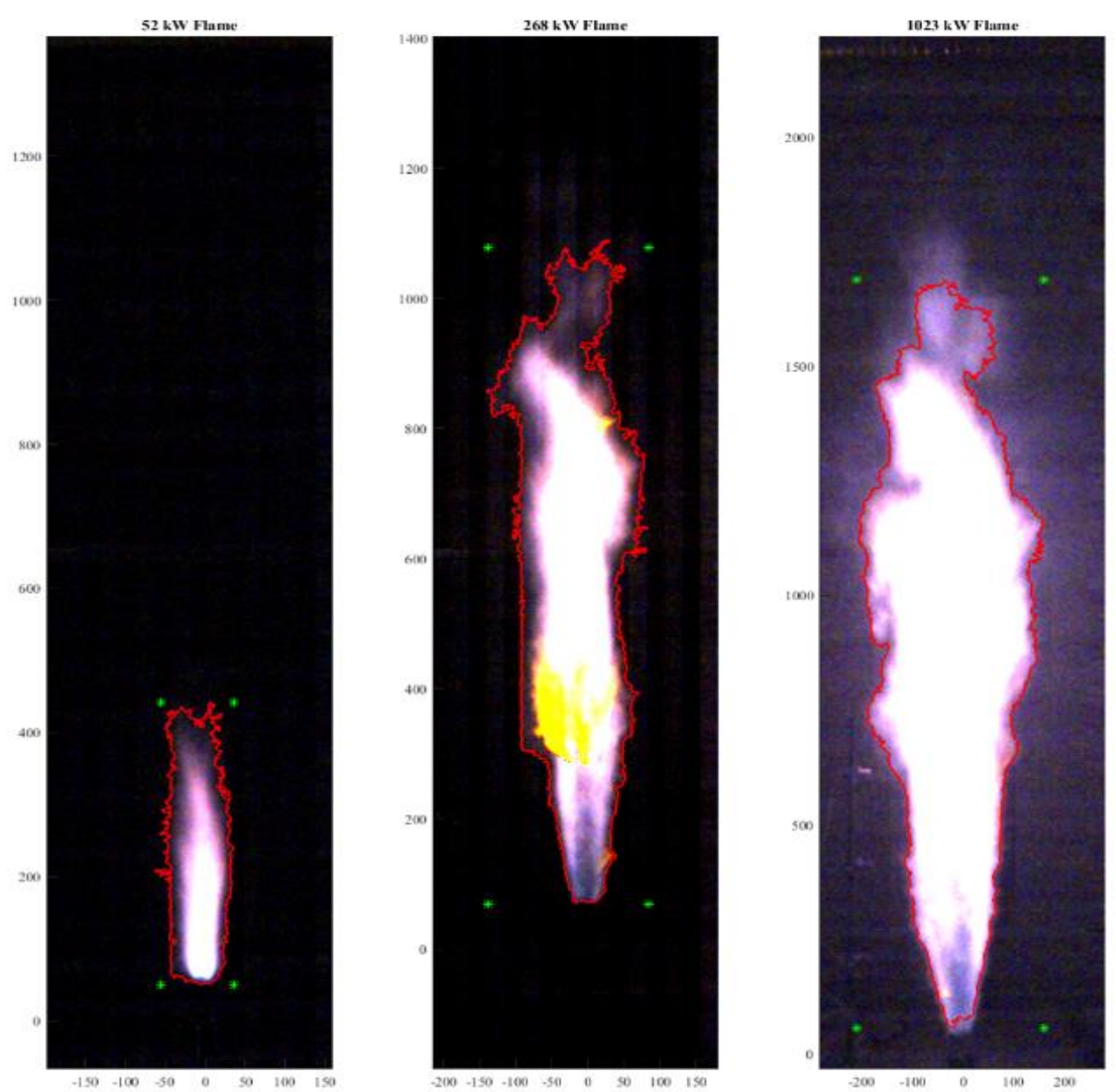

Figure 10. Three different sized hydrogen jet flames with contours of the calculated flame (red contours). The units are $\mathrm{mm}$ from exit nozzle.

The image-processing tool is explained in subsequent steps. The method used was iterative and the intermediate values are marked with an asterisk $(*)$.

\section{Step 1.}

Read RAWW image from image sequence (movie). From the camera, the movies are built up by 16 bits images. The images are built up by a string of 16 bits values for every color and pixel. For every movie, a header file tells how to read the string.

\section{Step 2}

The color image was converted into three monochrome images or channels,

$$
I_{R G B}=\left(I_{R}, I_{G}, I_{B}\right) \text {. }
$$

The monochrome 16 bits images was converted into numerical values (double). Since the different color channels showed no significant difference, the image was represented using

$$
I=I_{R}
$$

Step 3

The image was divided into flame and background using

$$
I= \begin{cases}F & : I_{i, j} \geq t \\ B & : I_{i, j}<t\end{cases}
$$

Where $F$ is the flame and $B$ is the background. $t$ is the threshold intensity value that separate the flame from the background. The value $t$ was not known and could be found iteratively.

\section{$\underline{\text { Step } 4}$}

An initial value for $\mathrm{t}$ was found by using

$$
t^{*}=\overline{S_{B}}+2 \cdot \operatorname{std}\left(S_{B}\right)
$$

where $S_{B}$ is a small area selection of the background of the image $I$. This was not a critical step, just an initial guess.

\section{Step 5}

The objects was isolated by finding the objects with an intensity above the threshold value $t^{*}$ using Equation 5 . The largest object was assumed to be the flame $F^{*}$. The rest was background, $B^{*}$.

\section{Step 6}

The new background $B^{*}$ was used to find a new threshold value using 


$$
t^{*}=\overline{B^{*}}+3 \cdot \operatorname{std}\left(B^{*}\right) .
$$

Then the routine was repeated from Step 5 until the change in $t^{*}$ was small. The final value of $t^{*}$ was the threshold value $t$, separating the flame and background.

$\underline{\text { Step } 7}$

The properties for the flame was collected and stored as a numerical value. The same thing was done to all the imaged of the movies providing the variation by time.

Figure 10 show the average flame height of three experiments with all three colors used to determine the flame height.

\section{Discussion}

In this work, the main target was to find a method to determine the height of a hydrogen jet flame using imaging techniques. The height of the jet flame was considered to represent the size of the hydrogen flame.

Two separate image processing techniques was considered, one based on Wiens distribution and one on intensity.

The technique based on Wiens distribution did not give any representable result due to low radiation from the flame and the high level of noise in the background.

The technique based an intensity was used and was compared to visual considerations of the flame.

\section{Conclusion}

An image processing technique was developed to determine the flame height of a hydrogen jet flame. The technique was based on the flame intensity and gave good results compared to visual considerations.

\section{References}

W. Li, C. Lou, Y. Sun, H. Zhou. Estimation of radiative properties and temperature distributions in coal-fired boiler furnaces by a portable image processing system, Exp. Therm. Fluid Sci. 35(2), 416-421, 2011.

T. Mogi, H. Nishida, S. Horiguchi, Flame Characteristics of High-pressure Hydrogen Gas Jet, Proceedings of the HySafe International Conference on Hydrogen Safety, Pisa, Italy, 8-10 Sept 2005.

T. Trindade, A. Ferreira, E. Fernandes. Characterization of Combustion Chemiluminescence: An Image Processing Approach, Proc. Tec., 17, 194-201, 2014.

W. Yan, S. Zheng, H. Zhou, Experiments investigation on 2D distribution of soot temperature and volume fraction by image processing of visible radiation, Applied Thermal Engineering, Volume 124, Pages 1014-1022, 2017.

Y. Zhang, S. Hui, Y. Zhang. The Research of Flame Combustion Diagnosis System Based on Digital Image Processing, CSEE 2011, pp. 225 - 230, 2011. 